\title{
On the Decidability of Semi-Linearity for Semi-Algebraic Sets and its Implications for Spatial Databases (extended abstract)
}

\author{
Freddy Dumortier* \\ University of Limburg \\ fdumorti@luc.ac.be
}

\author{
Marc Gyssens* \\ University of Limburg \\ gyssens@charlie.luc.ac.be \\ Dirk Van Gucht ${ }^{\dagger}$ \\ Indiana University \\ vgucht@cs.indiana.edu
}

\author{
Luc Vandeurzen* \\ University of Limburg \\ IvdeurzeQluc.ac.bo
}

\section{Abstract}

Several authors have suggested to use first-order logic over the real numbers to describe spatial database applications. Geometric objects are then described by polynomial inequalities with integer coefficients involving the coordinates of the objects. Such geometric objects are called semi-algebraic sets. Similarly, queries are expressed by polynomial inequalities. The query language thus obtained is usually referred to as FO + poly.

From a practical point of view, it has been argued that a linear restriction of this so-called polynomial model is more desirable. In the so-called linear model, geometric objects are described by linear inequalities, and are called semilinear sets. The language of the queries expressible by linear inequalities is usually referred to as FO + linear.

As part of a general study of the feasibility of the linear model, we show in this paper that semi-linearity is decidable for semi-algebraic sets. In doing so, we point out important subtleties related to the type of the coefficients in the linear inequalities used to describe semi-linear sets. An important concept in the development of the paper is regularity, of which we point out the geometric significance. We show that the regular points of a semi-linear set can be computed in $\mathrm{FO}+$ linear.

The decidability of semi-linearity of semi-algebraic sets has an important consequence. It has been shown that it is undecidable whether a query expressible in $\mathrm{FO}+$ poly is linear, i.e., maps spatial databases of the linear model into spatial databases of the linear model. It follows now that, despite this negative result, there exists a syntactically definable language precisely expressing the linear queries expressible in $\mathrm{FO}+$ poly.

\footnotetext{
-Dept. WNI, University of Limburg, Universitaire Campus, B-3590 Diepenbeek, Belgium. Contact author is Luc Vandeurzen.

${ }^{\dagger}$ Computer Science Dept., Indiana University, Bloomington, IN 47405-4101, USA.
}

Permission to make digital/hard copies of all or part of this material for personal or classroom use is granted without fee provided that the copies are not made or distributed for profit or commercial advantage, the copyright notice, the title of the publication and its date appear, and notice is given that copyright is by permission of the ACM, Inc. To copy otherwise, to republish, to post on servers or to redistribute to lists, requires specific permission and/or fee

PODS ' 97 Tucson Arizona USA

Copyright 1997 ACM 0-89791-910-6/97/05 ..\$3.50

\section{Introduction}

Following the seminal work by Kanellakis, Kuper, and Revesz [12] on constraint query languages with polynomial constraints, various researchers have introduced geometric database models and query languages within this framework $[10,17]$. These researchers have studied the desirability of their models for database applications involving geometric data objects, as well as the expressiveness of the proposed geometric query languages. We adopt the formalism of [17], which we shall call the polynomial spatial database model, in which both geometric objects and queries are expressed using polynomial inequalities. Geometric objects described by polynomial inequalities are called semi-algebraic sets, and the query language using polynomial inequalities is referred to as $\mathrm{FO}+$ poly.

Recently, several authors $[1,2,4,10,12,13,23,24]$ discussed linear spatial database models which can be seen as linear restrictions of the polynomial database model. These linear models allow users to define relational databases, which may, besides conventional data, contain linear geometric data objects, which suffice for the majority of applications encountered in GIS, geometric modeling, and spatial and temporal databases $[16,18]$. Furthermore, data structures and algorithms have been developed to efficiently implement a wide variety of operations on these sets $[5,6,7,9$, 20]. Geometric objects described by linear inequalities are called semi-linear sets, and the restriction of FO + poly using only linear inequalities is referred to as $\mathrm{FO}+$ linear. Not all linear queries (i.e., mappings between spatial databases describable in the linear model) expressible in $\mathrm{FO}+$ poly can be described in $\mathrm{FO}+$ linear, howcver $[1,24]$.

In the context of our investigation of the feasibility of the linear spatial database model as a restriction of the polynomial database model $[23,24]$, we focus in this paper on the decidability of semi-linearity for semi-algebraic sets.

In order to give an accurate solution to this problem, we point out that there are two natural ways to define the linear spatial database model as a restriction of the polynomial database models. A minimalistic approach consists of restricting the degree of the polynomials used in the polynomial model, yielding semi-linear sets described by linear constraints with integer coefficients. In this model, semialgebraic polytopes are semi-linear only if their corner points have rational coordinates. If, on the other hand, we want all semi-algebraic polytopes to be semi-linear, we have to take a maximalistic approach which consists of considering 
linear constraints with algebraic coefficients. (Observe that an algebraic number can be described finitarily by a univariate polynomial and an interval with rational end points in which this polynomial has precisely one root.)

We first prove that semi-linearity for semi-algebraic sets is decidable in the maximalistic case, and we provide an FO + poly expression for the corresponding decision query. Next, we prove that semi-linearity for semi-algebraic sets is decidable in the minimalistic case, too. However, we also prove that in this case there is no $\mathrm{FO}+$ poly expression for the corresponding decision query. Finally, we deduce from these decidability results that, both in the minimalistic and the maximalistic case, there exists a syntactically definable query language that precisely expresses all linear queries expressible in FO + poly. Earlier, three of the present authors showed that it is undecidable whether a $\mathrm{FO}+$ poly expression computes a linear query.

In the proof of the decidability results, the notion of regularity plays a key role. It allows the decomposition of a semi-algebraic set into so-called regular strata. In the case of semi-linear sets, the regular strata constitute a decomposition of the semi-linear set into linear components. We show that the query returning the regular points of a semi-linear set can be computed in $\mathrm{FO}+$ linear.

The remainder of this paper is organized as follows. Section 2 reviews the polynomial and linear spatial database models. Section 3 introduced the notion of regular stratification. Section 4 presents the key lemma of the paper. Section 5 contains the main decidability results. Section 6 , reflects on the implications of these results, and Section 7, finally, discusses the practical relevance of this work.

\section{Preliminaries}

We first review the polynomial model. The polynomial model is described using the first-order language of the ordered field of the real numbers $(R, \leq,+, x, 0,1)$, i.e., the language $(\leq,+, x, 0,1)$. The first-order formulae of this language are called real formulae. Seidenberg [21] and Tarski [22] showed that every real formula can effectively be transformed into a quantifier-free real formula. As a consequence, it is decidable whether a real sentence is valid in the ordered field of the real numbers. Every real formula $\varphi\left(x_{1}, \ldots, x_{n}\right)$ with free real variables among $x_{1}, \ldots, x_{n}$ defines a geometrical figure

$$
\left\{\left(x_{1}, \ldots, x_{n}\right) \mid \varphi\left(x_{1}, \ldots, x_{n}\right)\right\}
$$

in $n$-dimensional Euclidean space $\mathrm{R}^{n}$. Point sets defined in this way are called semi-algebraic sets.

A spatial database scheme $\mathcal{S}$ is a finite set of relation names. Each relation name $R$ has a type which is a pair of natural numbers $[m, n]$, where $m$ denotes the number of nonspatial columns and $n$ the dimension of the single spatial column of $R$. A database scheme has type $\left[m_{1}, n_{1}, \ldots, m_{k}, n_{k}\right]$ if the scheme consists of relation names, say $R_{1}, \ldots, R_{k}$, respectively of type $\left[m_{1}, n_{1}\right], \ldots,\left[m_{k}, n_{k}\right]$.

A syntactic spatial database instance is a mapping $\mathcal{I}$ assigning to each relation name $R$ of a database scheme $\mathcal{S}$ a syntactic spatial relation $\mathcal{I}(R)$ of the same type. A syntactic spatial relation of type $[m, n]$ is a finite set of tuples of the form $\left(v_{1}, \ldots, v_{m} ; \varphi\left(x_{1}, \ldots, x_{n}\right)\right)$, with $v_{1}, \ldots, v_{m}$ non-spatial values of some domain $U$, and $\varphi\left(x_{1}, \ldots, x_{n}\right)$ a real formula with $n$ free variables.

The semantics of a syntactic database instance $\mathcal{I}$ over a database scheme $\mathcal{S}$ is the mapping $I$ assigning to each relation name $R$ in $\mathcal{S}$ the semantic spatial relation $I(\mathcal{I}(R))$.
Given a syntactic spatial relation $r$, the semantic spatial relation $I(r)$ is defined as

$$
\bigcup_{t \in r}\left(\left\{\left(t . v_{1}, \ldots, t . v_{m}\right)\right\} \times\left\{\left(x_{1}, \ldots, x_{n}\right) \mid t . \varphi\left(x_{1}, \ldots, x_{n}\right)\right\}\right),
$$

a-possibly infinite-subset of $\mathbf{U}^{m} \times \mathbf{R}^{n}$.

Example 2.1 The example in Figure 1 shows a spatial database containing geographical information about Belgium. $\square$

In the polynomial model, we consider a query of signature $\left[m_{1}, n_{1}, \ldots, m_{k}, n_{k}\right] \rightarrow[m, n]$ to be a mapping from instances of a spatial database scheme of type $\left[m_{1}, n_{1}, \ldots\right.$, $\left.m_{k}, n_{k}\right]$ to instances of a spatial database scheme of type $[m, n]$ that can be regarded in a consistent way both at the syntactic and semantic level, and is computable at the syntactic level.

In this context, we define the query language $\mathrm{FO}+$ poly as the language obtained by adding to the language of real formulae the following: (i) a totally ordered infinite set of variables called non-spatial variables, disjoint from the set of real variables, (ii) atomic formulae of the form $v_{1}=v_{2}$, with $v_{1}$ and $v_{2}$ non-spatial variables, (iii) atomic formulae of the form $R\left(v_{1}, \ldots, v_{m} ; x_{1}, \ldots, x_{n}\right)$, with $v_{1}, \ldots, v_{m}$ non-spatial variables, $x_{1}, \ldots, x_{n}$ real variables, and $R$ a relation name of type $[m, n]$, and finally (iv) universal and existential quantification of non-spatial variables. A query of signature $\left[m_{1}, n_{1}, \ldots, m_{k}, n_{k}\right] \rightarrow[m, n]$ is definable in FO+poly if there exists an FO + poly formula $\varphi$ with $m$ free value variables and $n$ free real variables such that, for every input database instance of signature $\left[m_{1}, n_{1}, \ldots, m_{k}, n_{k}\right]$, $\left\{\left(v_{1}, \ldots, v_{m} ; x_{1}, \ldots, x_{n}\right) \mid \varphi\left(v_{1}, \ldots, v_{m}, x_{1}, \ldots, x_{n}\right)\right\}$ evaluates to the corresponding output database, which is of type $[m, n]$.

In this paper, the non-spatial part of a spatial database will play no role, and will therefore no longer be considered. In the same spirit, only purely spatial queries will be considered, i.e., queries of signature $\left[0, n_{1}, \ldots, 0, n_{k}\right] \rightarrow[0, n]$.

Example 2.2 Assuming that $S$ is a relation of type $[0,2]$, i.e., a semi-algebraic set in the plane, the $\mathrm{FO}+$ poly-formula

$$
\begin{gathered}
\left(\exists x_{1}\right)\left(\exists y_{1}\right)\left(\exists x_{2}\right)\left(\exists y_{2}\right)\left(\exists x_{3}\right)\left(\exists y_{3}\right)(\exists \lambda)(\exists \mu)(\exists \nu) \\
\left(S\left(x_{1}, y_{1}\right) \wedge S\left(x_{2}, y_{2}\right) \wedge S\left(x_{3}, y_{3}\right) \wedge\right. \\
\lambda \geq 0 \wedge \mu \geq 0 \wedge \nu \geq 0 \wedge \lambda+\mu+\nu=1 \wedge \\
\left.x=\lambda x_{1}+\mu x_{2}+\nu x_{3} \wedge y=\lambda y_{1}+\mu y_{2}+\nu y_{3}\right) .
\end{gathered}
$$

defines the convex-closure query of signature $[0,2] \rightarrow[0,2]$ which associates with $S$ its convex closure ${ }^{1}$.

From the polynomial model, a linear spatial database model can be obtained by only considering real formulae containing linear polynomials. There are two natural ways to achieve this restriction:

1. a minimalistic approach, in which only the degree of the polynomials involved is restricted to 1 , as a consequence of which all the linear polynomials considered have integer coefficients. The real formulae to which this restriction gives rise will be called Z-linear formulae, and the semi-algebraic sets that can be defined with them Z-semi-linear sets. Linear queries in

\footnotetext{
${ }^{1}$ Let $S \subseteq \mathbf{R}^{n}$. The convex closure of $S$ is the smallest convex set of $\mathbf{R}^{n}$ containing $S$.
} 


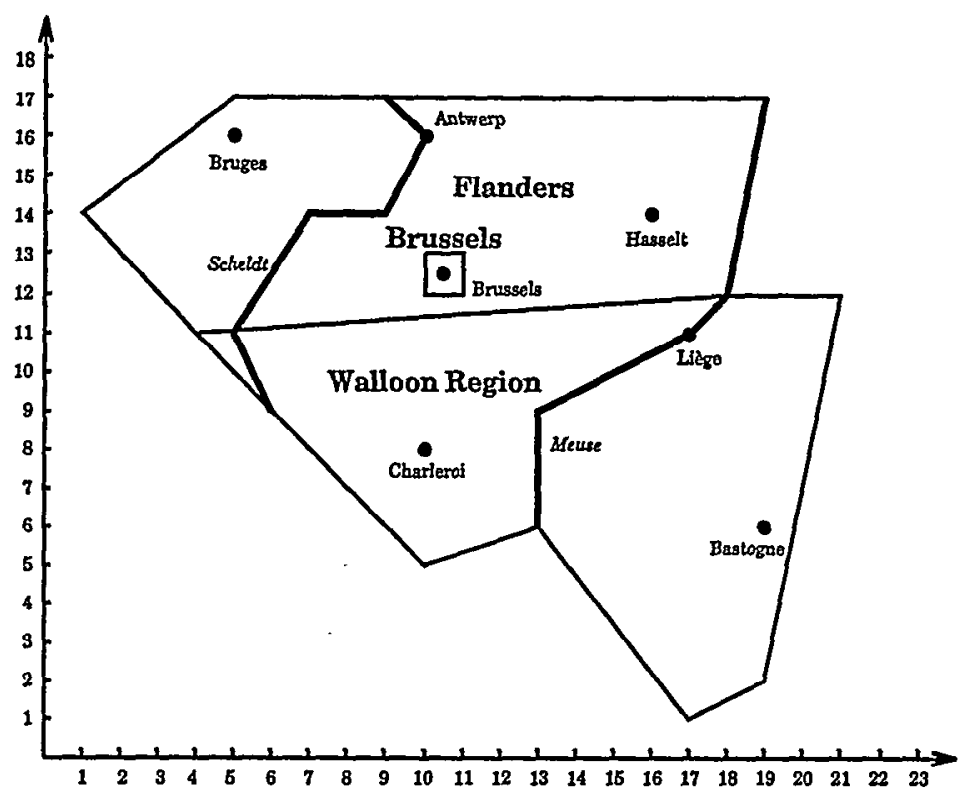

Regions

\begin{tabular}{|l|l|}
\hline Name & \multicolumn{1}{|c|}{ Geometry } \\
\hline Brussels & $(y \leq 13) \wedge(x \leq 11) \wedge(y \geq 12) \wedge(x \geq 10)$ \\
Flanders & $(y \leq 17) \wedge(5 x-y \leq 78 \wedge(x-14 y \leq-150) \wedge(x+y \geq 45) \wedge$ \\
Walloon Region & $(3 x-4 y \geq-53) \wedge(\neg(y \leq 13) \wedge(x \leq 11) \wedge(y \geq 12) \wedge(x \geq 10)))$ \\
& $((x-14 y \geq-15) \wedge(y \leq 12) \wedge(19 x+7 y \leq 375) \wedge(x-2 y \leq 15) \wedge$ \\
& $(5 x+4 y \geq 89) \wedge(x \geq 13)) \vee((-x+3 y \geq 5) \wedge(x+y \geq 45) \wedge$ \\
& $(x-14 y \geq-150) \wedge(x \geq 13))$ \\
\hline
\end{tabular}

Cities

\begin{tabular}{|l|l|}
\hline Name & \multicolumn{1}{|c|}{ Geometry } \\
\hline Antwerp & $(x=10) \wedge(y=16)$ \\
Bastogne & $(x=19) \wedge(y=6)$ \\
Bruges & $(x=5) \wedge(y=16)$ \\
Brussels & $(x=10.5) \wedge(y=12.5)$ \\
Charleroi & $(x=10) \wedge(y=8)$ \\
Hasselt & $(x=16) \wedge(y=14)$ \\
Lidge & $(x=17) \wedge(y=11)$ \\
\hline
\end{tabular}

Rivers
\begin{tabular}{|l|l|}
\hline Name & Geometry \\
\hline Meuse & $((y \leq 17) \wedge(5 x-y \leq 78) \wedge(y \geq 12)) \vee$ \\
& $((y \leq 12) \wedge(x-y=6) \wedge(y \geq 11)) \vee$ \\
& $((y \leq 11) \wedge(x-2 y=-5) \wedge(y \geq 9)) \vee$ \\
Scheldt & $((y \leq 9) \wedge(x=13) \wedge(y \geq 6))$ \\
& $((y \leq 17) \wedge(x+y=26) \wedge(y \geq 16)) \vee$ \\
& $((y \leq 16) \wedge(2 x-y=4) \wedge(y \geq 14)) \vee$ \\
& $((x \leq 9) \wedge(x \geq 7) \wedge(y=14)) \vee$ \\
& $((y \leq 14) \wedge(-3 x+2 y=7) \wedge(y \geq 11)) \vee$ \\
& $((y \leq 11) \wedge(2 x+y=21) \wedge(y \geq 9))$ \\
\hline
\end{tabular}

Figure 1: Example of a (linear) spatial database.

the context of Z-semi-linear sets will be called Z-linear and the corresponding restriction of $\mathrm{FO}+$ poly will be denoted $\mathrm{FO}+$ linear-Z. It turns out that semi-algebraic polytopes $^{2}$ are $\mathbf{Z}$-semi-linear only if their corner points have rational coefficients; and

2. a maximalistic approach, in which the linear polynomials considered may have arbitrary algebraic coefficients. For distinction, we speak about A-linear formulae, A-semi-linear sets, A-linear queries, and the query language FO + linear-A. It turns out that all semi-algebraic polytopes are A-semi-linear.

For use in the proof of the main result, we also consider $R$ linear formulae, in which the coefficients may be arbitrary real numbers, and, correspondingly, R-semi-linear sets. Every polytope is $\mathbf{R}$-semi-linear. Similarly, we consider $\mathbf{R}$-real

${ }^{2} \mathrm{~A}$ polytope is the convex closure of a finite set of points. formulae, in which the coefficients may be arbitrary real numbers, and, correspondingly, R-semi-algebraic sets.

Queries of signature $\left[m_{1}, n_{1}, \ldots, m_{k}, n_{k}\right] \rightarrow[0,0]$ are called Boolean queries, because the sets $\{(0)\}$ and \{\} can be seen as encoding the truth values true and false, respectively. Since both these sets are semi-linear, every Boolean query induces a linear query (in any of the approaches considered).

Example 2.3 The following FO + linear- $\mathrm{Z}$ formula defines a Boolean query (and hence a linear query in any of tho approaches considered) of signature $[0,2] \rightarrow[0,0]$ deciding whether $S$ is convex:

$$
\begin{array}{r}
\left(\forall x_{1}\right)\left(\forall y_{1}\right)\left(\forall x_{2}\right)\left(\forall y_{2}\right)\left(\forall x_{3}\right)\left(\forall y_{3}\right)\left(S\left(x_{1}, y_{1}\right) \wedge S\left(x_{2}, y_{2}\right) \wedge\right. \\
2 x_{3}=x_{1}+x_{2} \wedge 2 y_{3}=y_{1}+y_{2} \Rightarrow S\left(x_{3}, y_{3}\right) .
\end{array}
$$

Observe that the convex-closure query (Example 2.2) induces a linear query (in any of the approaches considered), which was shown to be unexpressible in FO+linear-Z [24]. 
Throughout the paper, we use vector notation to denote points. In this notation, equalities and inequalities of formulae should be interpreted coordinate-wise. Hence, $\rightarrow(\vec{p}=\overrightarrow{0})$ indicates that $\vec{p}$ is not the origin of the coordinate system, whereas $\vec{p} \neq \overrightarrow{0}$ denotes that none of the coordinates of $\vec{p}$ equals 0 . Finally, $\vec{p} \cdot \vec{q}$ denotes $p_{1} q_{1}+\cdots+p_{n} q_{n}$.

\section{Regular stratification}

The notions of a regular point and regular stratification of an R-semi-algebraic set $[3,8,15,25]$ play a key role in the technical development of this section.

Intuitively, a regular point of an $\mathbf{R}$-semi-algebraic set is a point of that set in which, locally, i.e., in some neighborhood of that point, the set looks like an algebraic variety (which can be described by equations only) which has a tangent space in $\vec{p}$.

Example 3.1 Consider the semi-algebraic set

$$
\begin{array}{r}
S=\left\{(x, y) \mid x^{2}+(y-1)^{2} \leq 9 \vee\right. \\
(y=1 \wedge 3 \leq x \leq 5) \vee(x=5 \wedge y=5)\}
\end{array}
$$

in the plane, shown in Figure 2, which consists of a closed disk with a closed line segment attached to it at the point $(3,1)$ and an isolated point.

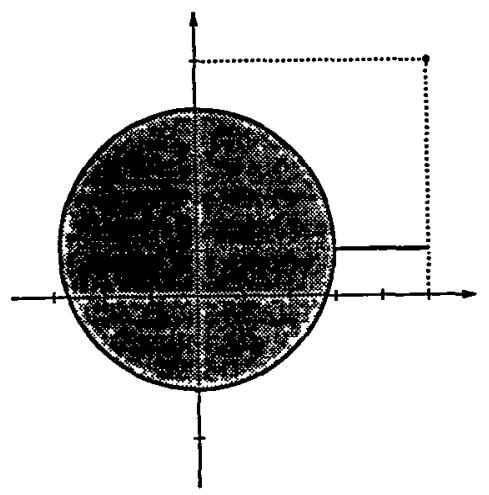

Figure 2: The semi-algebraic set of Example 3.1.

The regular points of $S$ are the points in the interior of the disk, the points of the open line segment, and the isolated points. In a small enough neighborhood of a point in the interior of the disk, the set $S$ looks like the whole plane, which happens to be also the tangent space in that point. In a small enough neighborhood of the a point in the open line segment, the set $S$ looks like the line $y=1$, which happens to be also the tangent space in that point. Finally, in a small enough neighborhood of the isolated point, the $S$ looks like that isolated point, which happens to be its own tangent space. In any neighborhood of a point on the boundary of the disk or the other end point of the line segment the $S$ does not look like an algebraic variety, and, therefore, these points are not regular.

We now formalize the intuition given above.

Definition 3.2 Let $S$ be an R-semi-algebraic set of $\mathbf{R}^{n}$ and let $\vec{p}$ be a point of $S$. The point $\vec{p}$ is a regular point of $S$ if there exists a neighborhood $V$ of $\vec{p}$, and polynomials with real coefficients $P_{1}, \ldots, P_{k}$ in $n$ real variables such that ${ }^{3}$

$$
\frac{\mathrm{d} P_{1}}{\mathrm{~d} \vec{x}}(\vec{p}), \ldots, \frac{\mathrm{d} P_{k}}{\mathrm{~d} \vec{x}}(\vec{p})
$$

are linearly independent and $S \cap V=\left\{\vec{x} \in V \mid P_{1}(\vec{x})=\right.$ $\left.\cdots=P_{k}(\vec{x})=0\right\}$.

If $S$ and $\vec{p}$ satisfy the above conditions, then, locally around $\vec{p}, S$ is an $n-k$-dimensional algebraic variety which has an $n-k$-dimensional tangent space at $\vec{p}$, defined by the system of $k$ linear equations

$$
\frac{\mathrm{d} P_{1}}{\mathrm{~d} \vec{x}}(\vec{p}) \cdot \vec{x}=0, \ldots, \frac{\mathrm{d} P_{k}}{\mathrm{~d} \vec{x}}(\vec{p}) \cdot \vec{x}=0 .
$$

We say that $S$ has dimension $n-k$ in $\vec{p}$ and call the maximum of these numbers the overall dimension of $S$, denoted $\operatorname{dim}(S)$.

Now, let $S$ be an R-semi-algebraic set, and let $\operatorname{Reg}(S)$ be the set of those regular points of $S$ in which $S$ has dimension $\operatorname{dim}(S)$. It is well-known that the connected components of $\operatorname{Reg}(S)$ are $\mathrm{R}$-semi-algebraic $[8,15]$. These are called regular strata. To $S-\operatorname{Reg}(S)$, which is again $\mathrm{R}$-semi-algebraic and of strictly lower dimension than $S$, we apply the same procedure, until no more points are left. In this way, we obtain a decomposition of $S$ in regular strata. Each R-semi-algebraic set has only a finite number of regular strata.

Example 3.3 Consider again the semi-algebraic set $S$, defined in Example 3.1, and shown in Figure 2. In Example 3.1, we observed that the set of regular points of $S$ consists of the open disk, the open line segment, and the isolated point. Only in the points of the open disk does $S$ have dimension 2. They form the only stratum in the first layer of the regular stratification. The complement of the first layer with respect to $S$, say $S^{1}$, consists of the circle, the closed line segment attached to it, and has overall dimension 1 . Its set of regular points consists of the circle from which the left end point of the attached line segment has been removed, the open line segment, and the isolated point. Only in the regular points of the circle and the line segment does $S^{1}$ have dimension 1. The circle from which the left end point of the attached line segment has been removed and the open line segment are therefore the 2 strata of the second layer of the regular stratification. Each of the remaining 3 points constitute a stratum in the third and final layer of the regular stratification of $S$.

If $S$ is an R-semi-linear set, then, locally around a regular point $\vec{p}, S$ will coincide with its tangent space at $\vec{p}$. This observation leads us to the following result (proof omitted):

Lemma 3.4 Let $S$ be an $\mathbf{R}$-semi-linear set of $\mathbf{R}^{n}$. The $\mathrm{FO}+$ linear $-\mathbf{Z}$ expression

$$
\begin{aligned}
& S(\vec{x}) \wedge(\exists \vec{\varepsilon})(\vec{\varepsilon} \neq \overrightarrow{0} \wedge(\forall \vec{y})(\forall \vec{z})(S(\vec{y}) \wedge \\
& \vec{x}-\vec{\varepsilon} \leq \vec{y} \leq \vec{x}+\vec{\varepsilon} \wedge S(\vec{z}) \wedge \vec{x}-\vec{\varepsilon} \leq \vec{z} \leq \vec{x}+\vec{\varepsilon} \Rightarrow \\
& (\exists \vec{u})(2 \vec{u}=\vec{y}+\vec{z} \wedge S(\vec{u})) \wedge(\exists \vec{v})(\vec{v}=2 \vec{x}-\vec{y} \wedge S(\vec{v})))) .
\end{aligned}
$$

defines the Z-linear query returning the regular points of $S$.

In previous work, three of the present authors [24] showed that the dimension query is expressible in FO + linear-Z. Using this result, Lemma 3.4 can be sharpened, as follows (proof omitted).

\footnotetext{
${ }^{3}$ For a function $f: R^{n} \rightarrow R$, and a point $\vec{p}$ of $R^{n}, \frac{d f}{d}(\vec{p})$ is defined as $\left(\frac{\partial f}{\partial x_{1}}(\vec{p}), \ldots, \frac{\theta f}{\partial x_{n}}(\vec{p})\right)$.
} 
Proposition 3.5 Let $S$ be an R-semi-linear set of $\mathbf{R}^{n}$. There exists an FO + linear-Z expression computing the $\mathrm{Z}$ linear query returning those regular points in which $S$ has dimension $\operatorname{dim}(S)$.

Hence, the subsequent layers of regular points encountered during a regular stratification of a semi-linear set are again semi-linear (in any of the approaches considered) and can effectively be computed (if the set is A- or Z-semilinear).

We now give two examples of the regular stratification of a semi-linear set.

Example 3.6 Consider the semi-linear set

$$
\begin{aligned}
& S=\{(x, y, z) \mid(0 \leq x \leq 3 \wedge 0 \leq y \leq 3 \wedge 0 \leq z \leq 3) \vee \\
& (3 \leq x \leq 5 \wedge y=1 \wedge z=0) \vee(x=5 \wedge y=5 \wedge z=0)\}
\end{aligned}
$$

in three-dimensional space, shown in Figure 3, which consists of a closed filled cube with a closed line segment attached to it at the point $(3,1,0)$ and an isolated point.

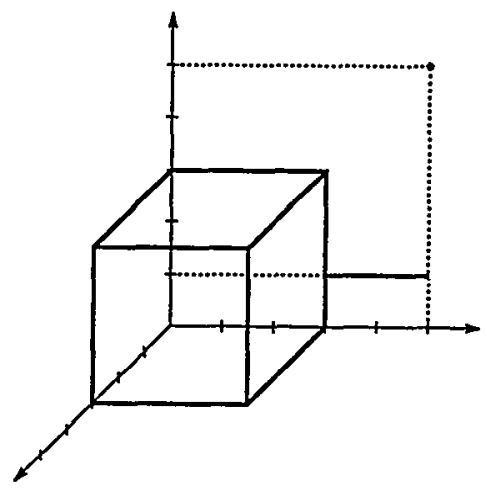

Figure 3: The semi-linear set of Example 3.6.

The set of regular points of $S$ consists of the open cube, the open line segment, and the isolated point. Only in the points of the open cube does $S$ have dimension 3. They form the only stratum in the first layer of the regular stratification. The complement of the first layer with respect to $S$, say $S^{1}$, consists of the faces of the cube, the closed line segment attached to it, and the isolated point, and has overall dimension 2. Its set of regular points consists of the 6 open faces of the cube, the open line segment, and the isolated point. Only in the points of the open faces does $S^{1}$ have dimension 2. The open faces of the cube are therefore the 6 strata of the second layer of the regular stratification. The complement of this second layer with respect to $S^{1}$, say $S_{2}$, consists of the edges of the cube, the line segment attached to it, and the isolated point, and has overall dimension 1. Its set of regular points consists of the 12 open edges of the cube with the exception of the point $(3,1,0)$, the open line segment, and the isolated point. Only in the points of the open edges with exception of the point $(3,1,0)$ and in the points of the open line segment does $S^{2}$ have dimension 1 . The two open line segments in which the point $(3,1,0)$ divides one of the open edges of the cube, the 11 remaining edges, and the line segment are therefore the 14 strata of the third layer of the regular stratification. The remaining 8 corner points, the point in which line segment meets the cube, and the isolated point each constitute a stratum in the fourth and final layer of the regular stratification of $S$.
Example 3.7 Consider the semi-linear set

$$
\begin{array}{r}
S=\{(x, y, z) \mid(3 x-y>2 \wedge y>1 \wedge x+2 y<10) \wedge \\
\neg(x \leq 3 \wedge y \geq 2 \wedge x \geq y) \\
\neg(1 \leq y \leq 3 \wedge x=3)
\end{array}
$$

in the plane, shown in Figure 4, which consists of an open triangle, out of which a closed triangle and a closed line segment have been cut out.

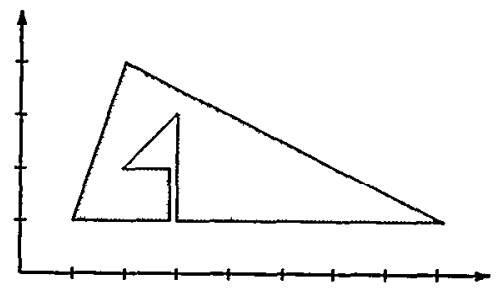

Figure 4: The semi-linear set of Example 3.7.

Each point of $S$ is regular, whence $S$ itself is the only regular stratum in the regular stratification of $S$.

\section{Key lemma}

To obtain the results announced earlier, we define the following property for point sets, called Property SL:

Definition 4.1 Let $S \subseteq \mathbf{R}^{n}$. We say that $S$ satisfies Property $S L$ if for every point $\vec{p}$ of the topological closure $\vec{S}$ of $S$, there exists a neighborhood $V$ of $\vec{p}$ such that, for every point $\vec{q}$ of $V$

1. if $\vec{q}$ is in $S$, then all points on the open line segment between $\vec{p}$ and $\vec{q}$ are also in $S$; and

2. if $\vec{q}$ is not in $S$, then none of the points on the open line segment between $\vec{p}$ and $\vec{q}$ is in $S$.

We claim that an $\mathbf{R}$-semi-algebraic set is $\mathbf{R}$-semi-lincar if and only if it has Property SL. Obviously, each R-somilinear set satisfies Property SL. Before proving the convorse, we first give an example illustrating that non-R-semi-linear sets do not have Property SL.

Example 4.2 Consider $S_{1}=\{(x, y) \mid x \geq 0 \wedge y \geq 0 \wedge$ $\left.x^{2}+y^{2}=4\right\}$ (Figure 5 , left) and $S_{2}=\{(x, y) \mid 0 \leq x \leq$ $\left.3 \wedge 0 \leq y \leq 3 \wedge x^{2}+y^{2} \neq 4\right\}$ (Figure 5, right). The set $S_{1}$ is a quarter circle, and the set $S_{2}$ is a square with a quarter circle cut out. Both are non-semi-linear semi-algebraic sets. The set $S_{1}$ fails Property SL, since no open line segment connecting two different points of $S_{1}$ is contained within $S_{1}$. The set $S_{2}$ fails Property SL, since each open line segment connecting two different points of the cut-out quarter circle meets $S_{2}$. Observe that the cut-out quarter circle belonga to the topological closure $\bar{S}_{2}$ of $S_{2}$.

We now prove our key lemma. The proof technique uses regular decomposition and is of interest in its own right.

Proposition 4.3 Let $S$ be an R-semi-algebraic set. The set $S$ is $\mathbf{R}$-semi-linear if and only if it has Property $S L$.

Proof. We provide a rough sketch of the proof of the "if."

First, we show that the class of R-semi-algebraic sets satisfying Property SL is closed under finite union and intersection, difference, and topological closure. Next, we consider the strata of the first regular layer of $S$, say $S_{1}, \ldots, S_{r}$. 

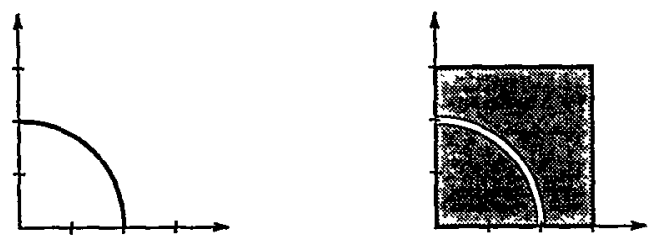

Figure 5: The non-semi-linear semi-algebraic sets of Example 4.2.

It is shown that $S_{1}, \ldots, S_{r}$ are open with respect to their affine supports ${ }^{4}$ and satisfy Property SL. As a consequence, $S-\bigcup_{i=1}^{r} S_{i}$ satisfies Property SL, whence the same reasoning can be reiterated on all subsequent regular layers. Thus, each regular stratum of $S$ is open with respect to its affine support and satisfies Property SL. To prove Proposition 4.3, it suffices to show that each regular stratum individually is R-semi-linear.

Thus, let $S_{l}$ be a stratum in the regular stratification of $S$. Since the topological closure $\bar{S}_{l}$ of $S_{l}$ is an R-semialgebraic set satisfying Property SL, so is $\partial S_{l}=\vec{S}_{l}-S_{l}{ }^{5}$. Thus we can repeat the above reasoning on $\partial S_{l}$. Let $T_{0}=S_{l}$ and let $T_{1}, \ldots, T_{s}$ be the decomposition of $\partial S_{l}$ obtained. Each $T_{i}, 0 \leq i \leq s$, is open with respect to its affine support, say $A_{i}$. Each $A_{i}$ is the intersection of hyperplanes $H_{1}^{i}, \ldots, H_{t_{i}}^{i}, 0 \leq t_{i} \leq n$. Each hyperplane $H_{j}^{i}, 0 \leq i \leq s$, $1 \leq j \leq t_{i}$, partitions $\mathbf{R}^{n}$ into two open half-spaces and their separating hyperplane, all three of which are obviously $\mathrm{R}$-semi-linear. Of all these partitions, we now consider the coarsest common refinement. The classes of this last partition are finite intersections of classes of the original partitions, and are therefore also R-semi-linear. Finally, it is shown that $S_{l}$ is the (finite) union of all the classes with which it has a non-empty intersection, whence $S_{l}$ is also $\mathbf{R}$-semi-linear.

We illustrate the proof of Proposition 4.3.

Example 4.4. In the first paragraph of the proof of Proposition 4.3 , a regular stratification of the set $S$ under consideration is obtained. This process has already been illustrated in Examples 3.3, 3.6, and 3.7. Then, each stratum is considered separately in the second paragraph of the proof.

To illustrate the second paragraph of the proof, we consider again Example 3.7, since, in this example, the set $S$ coincides with its only regular stratum.

In the next stage of the proof, we consider $\partial S=\vec{S}-S$, which is shown in Figure 6.

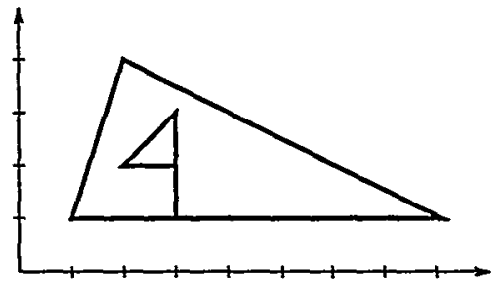

Figure 6: The set $\partial S$ of Example 4.4. .

When this figure is decomposed, the resulting regular stratification consists of the 7 special points and the 8 line

\footnotetext{
${ }^{4}$ Let $S \subseteq \mathrm{R}^{n}$. The affine support of $S$ is the smallest affine variety of $\mathrm{R}^{n}$ containing $S$.

${ }^{5}$ Here, $\partial S_{l}$ denotes the topological boundary of $S_{l}$ with respect to its affine support.
}

segments connecting them. Thus, the sequence $T_{0}, T_{1}, \ldots, T_{s}$ in the proof of Proposition 4.3 consists of $S$ itself, 8 line segments and 7 points, 16 point sets in total. The respective affine supports of these 16 sets are the entire plane, supporting $S$, the lines supporting the 8 line segments, and the 7 points, supporting themselves. According to the proof of Proposition 4.3, all 16 point sets under consideration here are open in their respective affine supports. Now, each of the 16 affine supports is an intersection of 0,1 , or more hyperplanes, which, in the two-dimensional plane, are lines. Indeed, the entire plane is the empty intersection of lines and a point is the intersection of two non-parallel lines. If we describe the 7 points by the affine supports of the line segments that meet in these points, then Figure 7, shows all the lines involved. These lines, together with the open half-planes they define, induce a partition of the entire plain, consisting of 12 points, 31 open line segments or half-lines, and 20 open regions. In Figure 7, these regions have been identified by numbers. By construction, all 63 members of the induced partition are semi-linear sets.

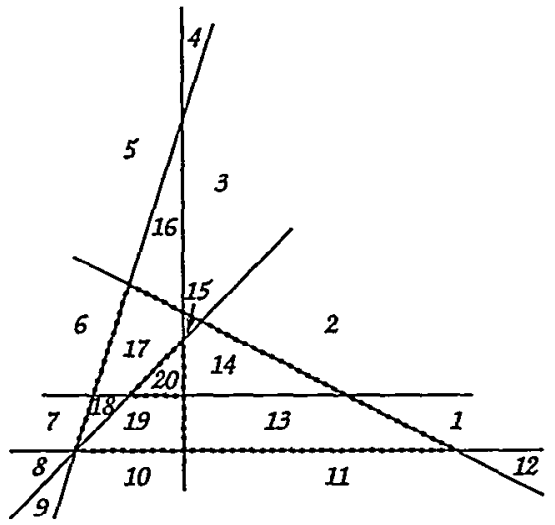

Figure 7: The lines needed to describe the affine supports of the regular strata in the decomposition of the set $\partial S$ of Example 4.4. The numbers indicate the open regions in the induced partition.

The closing argument of the proof of Proposition 4.3 is that $S$ is the union of some members of the induced partition, and, thereby, also semi-linear. Indeed do we see in this example that $S$ is the union of the open regions $13,14,15$, 17,18 , and 19, all of which are filled polygons, and the open intervals separating them.

\section{Decidability results}

Proposition 4.3 can be sharpened to our first main result:

Theorem 5.1 Let $S$ be a semi-algebraic set. The set $S$ is A-semi-linear if and only if it has Property SL. Moreover, A-semi-linearity of semi-algebraic sets is decidable.

The second statement in Theorem 5.1 follows from the first, because the corresponding Boolean decision query of type $[0, n] \rightarrow[0,0]$ can easily be expressed in FO + poly using Property SL, and the validity of real sentences in $R$ is decidable.

Unfortunately, the truth of the first statement is not revealed by the proof of Proposition 4.3, because Definition 3.2 of regular point does not specify anything regarding the type of the coefficients in the polynomials involved. Rather, we 
shall derive the first statement of Theorem 5.1 from Proposition 4.3 using the following two lemmas. The second one is stated more strongly than strictly required, in anticipation of the proof of Theorem 5.6.

Lemma 5.2 Every bounded R-semi-linear semi-algebraic set is A-semi-linear.

Proof. We provide a rough sketch of the proof.

Let $S$ be a bounded $\mathrm{R}$-semi-linear semi-algebraic set. It suffices to show that each regular stratum $S_{l}$ of $S$ is Asemi-linear. Therefore, let $T_{0}, \ldots, T_{s}$ be as in the proof of Proposition 4.3. For each $T_{i}, 1 \leq i \leq s$, let $A_{i}$ be the affine support of $T_{i}$. We show by induction on $\operatorname{dim}\left(T_{i}\right)$, the dimension of $T_{i}$, that each $T_{i}$ has the following Property $A C$ :

$A_{i}$, the affine support of $T_{i}$, is the intersection of hyperplanes which can be described by linear equations with algebraic coefficients.

The A-semi-linearity of $S_{l}$ then follows as in the last part of the proof of Proposition 4.3.

Now consider the following procedure. Let $S^{1}$ be the first layer in the regular stratification of $S$. By Proposition 3.5, this layer can be computed from $S$ in FO + linear-Z. Since $S$ is semi-algebraic, so are $S^{1}, S-S^{1}$, and $S^{1}-S^{1}$. Both $S-S^{1}$ and $S^{1}-S^{1}$ have strictly lower dimension than $S$. Repeat this procedure on $S-S^{1}$ and $\bar{S}^{1}-S^{1}$ independently, and let $S^{u}$ be the union of all the 0 -dimensional layers finally obtained. Thus $S^{u}$ is semi-algebraic. If $\operatorname{dim}\left(T_{i}\right)=0$, i.e., $T_{i}$ consists of a single point, then it can be seen that $T_{i} \subseteq$ $S^{u}$ (details omitted). Therefore, $T_{i}$ is also semi-algebraic, whence it can be described by a real formula. By the TarskiSeidenberg quantifier elimination theorem $[21,22]$, it follows that the coordinates of that point can be described using univariate polynomials. By definition, it follows that these coordinates must be algebraic, whence $T_{i}=A_{i}$ satisfies Property AC.

Now assume $\operatorname{dim}\left(T_{i}\right)=d, 1 \leq d \leq n$. Let

$$
\begin{aligned}
J=\left\{j \mid 1 \leq j \leq n \wedge \operatorname{dim}\left(T_{j}\right) \leq d-1 \wedge\right. \\
\left.T_{j} \text { is in the boundary of } T_{i}\right\} .
\end{aligned}
$$

Assume, for all $j$ in $J$, that $T_{j}$ has Property AC. Since $S$ is bounded, $\bar{S}_{l}$ and $T_{i}$ are also bounded. Hence, $A_{i}$, the affine support of $T_{i}$, is also the affine support of the boundary of $T_{i}$. Since the boundary of $T_{i}$ equals $\bigcup_{j \in J} T_{j}, A_{i}$ is also the affine support of $\bigcup_{j \in J} A_{j}$. Now, it readily follows from the inductive hypothesis that $T_{i}$ has property $\mathrm{AC}$.

Lemma 5.3 For each R-semi-linear semi-algebraic set $S$, there exists a positive integer $d$ that can effectively be computed such that, for the open filled cube $C(\overrightarrow{0}, d)$ with center $\overrightarrow{0}$ and edges $2 d$,

1. $S$ is A-semi-linear if and only if $S \cap C(\overrightarrow{0}, d)$ is A-semilinear, and

\section{2. $S$ is Z-semi-linear if and only if $S \cap C(\overrightarrow{0}, d)$ is Z-semi-} linear.

Proof. We provide a rough sketch of the proof.

We require that, for each affine variety $A$ which is the support of some regular stratum, there is a (possibly, but not necessarily, different) stratum in the same layer of the stratification which has $A$ as its affine support and which has a non-empty intersection with $C(\overrightarrow{0}, d)$. The set $D$ of all real numbers $d$ satisfying this requirement is non-empty, since there are only a finite number of affine varieties to be considered. Hence, $D$ is a half line. Moreover, the query returning $D$ on input $S$ can be described in FO+poly (details omitted). As a consequence, membership of $D$ is decidable. By enumeration, one can effectively find the smallest integor $d$ in $D$.

Since $C(\overrightarrow{0}, d)$ is Z-semi-linear, $S \cap C(\overrightarrow{0}, d)$ is A-semi-linear (Z-semi-linear) whenever $S$ is A-semi-linear (Z-semi-linear).

By construction, all the linear polynomials needed to describe $S$ as in the proof of Proposition 4.3 are also needed to describe $S \cap C(\overrightarrow{0}, d)$. Thus, conversely, $S$ is A-semi-linear (Z-semi-linear) whenever $S \cap C(\overrightarrow{0}, d)$ is A-semi-linear (Zsemi-linear).

From Lemmas 5.2 and 5.3 , it follows that every $\mathbf{R}$-somilinear semi-algebraic set is A-semi-linear. Theorem 5.1 is now readily deduced from Proposition 4.3.

By Theorem 5.1, A-semi-linearity of a semi-algebraic set is decidable. In view of the reasons for which semi-linear sots are most often preferred over general semi-algebraic sets, Z-semi-linearity is a far more interesting property than $A$ semi-linearity. Therefore, we next proceed to show that $\mathbf{Z}$. semi-linearity of a semi-algebraic set is decidable, too.

Lemma 5.4 Suppose an algebraic number is given by a univariate polynomial equation with integer coefficients and an open interval with rational endpoints which contains that algebraic number as only solution of the equation. It is decidable whether that algebraic number is rational.

Proof. It is an easily provable consequence of Eisenstein's irreducibility criterion that any rational root of a polynomial $a_{n} x^{n}+\cdots+a_{0}$ can be written as $r / s$ with $r$ and $s$ relatively prime, $r \mid a_{0}$, and $s \mid a_{n}$. (This result is called the rational root theorem in [19].) Hence, there are only a finite number of rational numbers for which the conditions defining the algebraic number have to be verified.

Lemma 5.5 It is decidable whether a bounded semi-algebraic set defined by a real formula is Z-semi-linear.

Proof. We provide a rough sketch of the proof.

Let $S$ be a bounded semi-algebraic set. First, we verify whether $S$ satisfies Property SL. If $S$ does not satisfy property SL, it is not R-semi-linear (Proposition 4.3), whence certainly not Z-semi-linear; else it is A-semi-linear (Theorem 5.1). In the latter case, we perform the construction explained in the proof of Lemma 5.2, leading to a 0 dimensional set $S^{\mathbf{u}}$. All the steps of this construction can be expressed in $\mathrm{FO}+$ linear- $\mathrm{Z}$.

Thus, if $S$ is Z-semi-linear, so is $S^{u}$, whence all the points constituting $S^{u}$ must have rational coordinates. Conversely, if all the points constituting $S^{u}$ have rational coordinates, an inductive proof along the lines of the proof of Lemma 5.2 can be given to show that all regular strata of $S$ are Z-semilinear, whence $S$ is.

By Lemma 5.4, it is decidable whether a semi-algebraic singleton set consists of a point with rational coordinates. $\square$

Theorem 5.6 It is decidable whether a semi-algebraic set defined by a real formula is Z-semi-linear.

Proof. Let $S$ be a semi-algebraic set. First, we verify whether $S$ satisfies Property SL. If $S$ does not satisfy property SL, it is not R-semi-linear (Proposition 4.3), whence 
certainly not Z-semi-linear; else it is A-semi-linear (Theorem 5.1). In the latter case, we compute a open full cube $C(\overrightarrow{0}, d)$ with center $\overrightarrow{0}$ and edges $2 d$ such that $S$ is Z-semilinear if and only if $S \cap C(\overrightarrow{0}, d)$ is Z-semi-linear (Lemma 5.3). By Lemma 5.5 , it is decidable whether $S \cap C(\overrightarrow{0}, d)$ is Z-semilinear.

However, the Boolean query deciding the Z-semi-linearity of a semi-algebraic set is not expressible in $\mathrm{FO}+$ poly.

Theorem 5.7 The Boolean query of type $[0, n] \rightarrow[0,0]$ deciding the Z-semi-linearity of a semi-algebraic set is not expressible in $\mathrm{FO}+$ poly.

Proof. Assume to the contrary that there exists a sentence $\sigma$ in the first-order language $(\leq, S,+, \times, 0,1)$, with $S$ an $n$-dimensional predicate, such that, for each possible interpretation of $S$ as a semi-algebraic set, $\sigma$ is true if and only if this interpretation is a $\mathbf{Z}$-semi-linear set of $\mathbf{R}^{n}$.

Now, let $x_{1}, \ldots, x_{n}$ be real variables not occurring in $\sigma$, and transform $\sigma$ into a real formula $\varphi\left(x_{1}, \ldots, x_{n}\right)$ by replacing each subformula $S\left(y_{1}, \ldots, y_{n}\right)$ in $\sigma$, with $y_{1}, \ldots, y_{n}$ bound variables, by the subformula $\left(x_{1}=y_{1} \wedge \ldots \wedge x_{n}=\right.$ $\left.y_{n}\right)$. Hence $\varphi\left(x_{1}, \ldots, x_{n}\right)$ evaluates to true if and only if the evaluation of $\left(x_{1}, \ldots, x_{n}\right)$ is a point with rational coordinates. Now, let $\psi(x)$ be the real formula $\varphi(x, \ldots, x)$. Then $\{x \mid \psi(x)\}$ is the set of all rational numbers, which is not semi-algebraic, a contradiction.

\section{Discussion}

We first discuss some philosophical consequences of our results.

A first consequence of the results in the previous section is that, whenever a real formula $\varphi(\vec{x})$ defines an Asemi-linear (a Z-semi-linear) set, an equivalent A-linear (Zlinear) formula can effectively be computed. First, verify Property SL to find out whether $\varphi(\vec{x})$ defines an A-semilinear set (Theorem 5.1). If necessary, verify whether $\varphi(\vec{x})$ defines a Z-semi-linear set (Theorem 5.6). In the case of a positive answer, enumerate all A-linear (Z-linear) formulae $\psi(\vec{x})$ and decide whether the real sentence $(\forall \vec{x})(\varphi(\vec{x}) \Leftrightarrow$ $\psi(\vec{x}))$ is true in R. Because $\varphi(\vec{x})$ is already known to define an A-linear (a Z-linear) set, such a formula $\psi(\vec{x})$ must be found.

A second consequence of the results in the previous section concerns A-linear and Z-linear queries. We recall the following result proved by three of the present authors [24]:

Theorem 6.1 It is undecidable whether an FO + poly-expression induces an A-linear (a Z-linear) query.

Our results show, however, that there exists a syntactically definable query language which expresses precisely the A-linear (Z-linear) queries expressible in FO + poly. Syntactically, this query language is just FO + poly. However, another semantics is given to $\mathrm{FO}+$ poly expressions. This semantics is obtained by modifying the standard output of FO + poly expression applied to some input database as follows: all semi-algebraic sets represented in this standard output that are not A-semi-linear (Z-semi-linear) are replaced by the empty set. With respect to the A-linear (Zlinear) queries expressible in $\mathrm{FO}+$ poly, the language thus obtained is sound (it returns linear outputs on linear inputs) as well as complete (it does not modify the standard semantics of linear FO + poly queries). Notice that this result does not contradict Theorem 6.1 , in the same way that the existence of a syntactically definable query language which is sound and complete for the domain-preserving calculus queries in the relational model does not contradict the undecidability of domain preservation for arbitrary calculus queries.

For A-linear queries, the above new semantics of an $\mathrm{FO}+$ poly expression can be expressed by an $\mathrm{FO}+$ poly expression in the standard way. As a consequence, there exists a recursively enumerable subset of $\mathrm{FO}+$ poly which expresses precisely the A-linear queries expressible in $\mathrm{FO}+$ poly, according to the standard semantics of FO + poly. Whether a similar result holds for the Z-linear queries is still open. (Because of Theorem 5.7, the argument used for A-linear queries fails for Z-linear queries).

Finally, the asymmetry between both decidability results (A-semi-linearity being expressible in $\mathrm{FO}+$ poly, and Zsemi-linearity being not expressible in $\mathrm{FO}+$ poly) and some of their consequences emphasize the necessity of properly distinguishing these two notions of semi-linearity.

\section{Practical relevance}

Of course, the query language complete for the A-linear (Zlinear) queries described in the previous section, is very artificial and not practically useful. Nevertheless, our results show that a A-linear-complete (Z-linear-complete) query language exists, and, therefore, show that is reasonable to search for a more practical such language.

Furthermore, the techniques developed to derive our results also have more immediate ramifications in that they can be used to show that certain Z-linear queries can be expressed in $\mathrm{FO}+$ linear-Z.

For example, the proof of Lemma 5.2 contains a procedure that, given a bounded semi-algebraic set $S$, computes a set of points, called $S^{u}$. Intuitively, these are the "key" points of $S$, from which $S$ can be "reconstructed." (As a matter of fact, this is what happens in the remainder of the proof of Lemma 5.2.) Recently, other researchers have also considered these "special points;" Grumbach and Kuper, e.g., call them significant points [14]. A special case is exhibited in the following example.

Example 7.1 Corners of a polygon.

Let $S$ be a closed filled polygon in the plane. Then $(S-\operatorname{Reg}(S))-\operatorname{Reg}(S-\operatorname{Reg}(S))$ is the set of all corner points of this polygon. By our results, this set can be computed in FO + linear-Z. This technique can of course be generalized to higher-dimensional simplices and higher-dimensional spaces.

It must be noted, moreover, that not only the 0-dimensional sets (i.e., the points) yielded by the procedure in the proof of Lemma 5.2 are of interest, but also, e.g., the 1dimensional sets. In computer graphics, the union of all this 1-dimensional sets is called the wire frame of $S$ [11], which is used to render 3-dimensional figures. A special case is exhibited in the following example.

\section{Example 7.2 Wire frame of a polyhedron.}

Let $S$ be a closed filled polyhedron in three-dimensional space. Then $(S-\operatorname{Reg}(S))-\operatorname{Reg}(S-\operatorname{Reg}(S))$ is the wire frame of $S$. As in Example 7.1, the wire frame can be computed in FO + linear-Z. Again, the computation of wire frames can be generalized to higher-dimensional simplices and higherdimensional spaces. 


\section{Acknowledgments}

The authors wish to thank Paul Purdom for interesting discussions and for his help in the search of related literature.

The authors also wish to thank the anonymous referees for their comments, which were very valuable for improving the presentation of this paper. One referee provided very detailed comments. In particular, he or she pointed out that the results in Section 5 can alternatively be obtained using definability results in logic, in a very elegant way. However, the authors decided not to incorporate this alternative method in the present version of the paper, because they feel they cannot possibly do justice to this referee's welltaken point within the inherent limitations of an extended abstract, also because the authors' present approach has some ramifications toward practical applications.

\section{References}

[1] F. Afrati, S. Cosmadakis, S. Grumbach, and G. Kuper, "Linear Versus Polynomial Constraints in Database Query Languages," in Proceedings 2nd Int'l Workshop on Principles and Practice of Constraint Programming (Rosario, WA), A. Borning, ed., in Lecture Notes in Computer Science, vol. 874, Springer-Verlag, 1994, pp. 181-192.

[2] M. Benedikt, G. Dong, L. Libkin, and L. Wong, "Relational expressive power of constraint query languages," in Proceedings 15th ACM SIGACT-SIGMOD-SIGART Symposium on Principles of Database Systems (Montreal, Canada), 1996, pp. 5-16.

[3] J. Bochnak, M. Coste, and M-F. Roy, Géométrie algébrique réelle, Springer-Verlag, Berlin-Heidelberg, 1987.

[4] A. Brodsky and Y. Kornatzky, "The LyriC Language: Querying Constraint Objects," in Proceedings Post-ILPS'g4 Workshop on Constraints and Databases (Ithaca, NY), 1994.

[5] I. Carlbom, "An Algorithm for Geometric Set Operations Using Cellular Subdivision Techniques," IEEE Computer Graphics and Applications, 7:5, 1987, pp. 4455.

[6] D.Q. Goldin and P.C. Kanellakis, "Constraint Query Algebras," Constraints Journal, 1, 1996, pp. 1-41.

[7] O. Günther, ed., Efficient Structures for Geometric Data Management, in Lecture Notes in Computer Science, vol. 337, Springer-Verlag, 1988.

[8] J. Heintz, T. Recio, and M.-F. Roy, "Algorithms in Real Algebraic Geometry and Applications to Computational Geometry," in Discrete and Computational Geometry: Papers from the DIMACS Special Year, J.E. Goodman, R. Pollack, and W. Steiger, eds., in DIMACS Series in Discrete Mathematics and Theoretical Computer Science, vol. 6, 1991, pp. 137-163.

[9] T. Huynh, C. Lassez, and J.-L. Lassez, "Fourier Algorithm Revisited," in Proceedings 2nd Int'l Conf. on Algebraic an Logic Programming, H. Kirchner and W. Wechler, eds., in Lecture Notes in Computer Science, vol. 463, Springer-Verlag, 1990, pp. 117-131.
[10] P.C. Kanellakis and D.Q. Goldin, "Constraint Programming and Database Query Languages," in Proceedings 2nd Conf. on Theoretical Aspects of Computer Software, M. Hagiya and J.C. Mitchell, eds., Lecture Notes in Computer Science, vol. 789, Springer-Verlag, 1994, pp. 96-120.

[11] A. Kemper and M. Wallrath, "An Analysis of Geometric Modeling in Database Systems," ACM Computing Surveys, 19:1, March 1987, pp. 47-91.

[12] P.C. Kanellakis, G.M. Kuper, and P.Z. Revesz, "Constraint Query Languages," Journal of Computer and System Sciences, 51:1, 1995, pp. 26-52.

[13] G. Kuper, "On the Expressive Power of the Relational Calculus with Arithmetic Constraints," in Proceedings 3rd International Conference on Database Theory (Paris, France), S. Abiteboul and P.C. Kanellakis, eds., in Lecture Notes of Computer Science, vol. 470 Springer-Verlag, pp. 202-214.

[14] G. Kuper, personal communications, Antwerp, December 3, 1996.

[15] T. Mostowski and E. Rannou, "Complexity of the Computation of the Canonical Whitney Stratification of an Algebraic Set in $\mathbf{C}^{n}, "$ in Proceedings 9th International Symposium on Applied Algebra, Algebraic Algorithms and Error-Correcting Codes (New Orleans, Louisiana, USA, October 1991), H.F. Mattson, T. Mora, and R.R.N. Rao, eds., in Lecture Notes in Computer Science, vol. 539, Springer Verlag, 1991, pp. 281-291.

[16] J. Nievergelt and M. Freeston, eds., Special issue on spatial data, Computer Journal, 37:1, 1994.

[17] J. Paredaens, J. Van den Bussche, and D. Van Gucht, "Towards a Theory of Spatial Database Queries," in Proceedings 13th ACM SIGACT-SIGMOD-SIGART Symposium on Principles of Database Systems (Minneapolis, Minnesota, USA), 1994. pp. 279-288.

[18] N. Pissinou, R. Snodgrass, R. Elmasri, I. Mumick, T. Özsu, B. Pernici, A. Segef. B. Theodoulidis, and U. Dayal, "Towards an Infrastructure for Temporal Databases," SIGMOD Record, 23:1, 1994, pp. 35-51.

[19] H. Pollard and H.G. Diamond. "The Theory of Algebraic Numbers." The Carus Mathematical Monographs 9, 2nd edition, The Mathematical Association of America, 1975, p. 41.

[20] L.K. Putnam and P.A. Subrahmanyam, "Boolean Operations on $n$-Dimensional Objects," IEEE Computer Graphics and Applications, 6:6, 1986, pp. 43-51.

[21] A. Seidenberg, "A New Decision Procedure for Elementary Algebra," Annals of Mathematics, 60, 1954, pp. 365-374.

[22] A. Tarski, "A Decision Method for Elementary Algebra and Geometry," University of California Press, Berkeley, California, 1951.

[23] L. Vandeurzen, M. Gyssens, and D. Van Gucht, "On the Desirability and Limitations of Linear Spatial Query Languages," in Proceedings 4th Symposium on Advances in Spatial Databases (Portland, Oregon, USA, August 1995), M. J. Egenhofer and J.R. Herring, eds., in Lecture Notes in Computer Science, vol. 951, Springer-Verlag, 1995, pp. 14-28. 
[24] L. Vandeurzen, M. Gyssens, and D. Van Gucht, "On Query Languages for Lincar Qucries Definable with Polynomial Constraints," in Proceedings Second International Conference on Principles and Practice of Constraint Programming (Cambridge, Massachusetts, USA, August 1996), E. Freuder, ed., in Lecture Notes in Computer Science, vol. 1118, Springer-Verlag, 1996, pp. $468-481$.
[25] H. Whitney, "Elementary Structure of Real Algebraic Varieties," Annals of Mathematics, 66, 1957, pp. 545556. 